\title{
An Enhanced Widely Linear CDMA Receiver With OQPSK Modulation
}

\author{
Arash Mirbagheri, Member, IEEE, Konstantinos N. Plataniotis, Senior Member, IEEE, and \\ Subbarayan Pasupathy, Life Fellow, IEEE
}

\begin{abstract}
This paper studies an enhanced widely linear (WL) receiver for direct-sequence code-division multiple-access (DS-CDMA) systems that employ aperiodic spreading sequences with offset quadrature phase-shift keying (OQPSK) modulation. The modulation scheme generates improper complex multiple-access interference (MAI) and necessitates the use of WL receivers. Focus is on bandlimited pulse shapes and the inherent cyclostationarity $(\mathrm{CS})$ of the uncoded received signal. The enhanced WL receiver replaces the conventional chip-matched filter with new filters that exploit the CS of the received signal through frequency shifting. The proposed WL receiver is shown to outperform the classical strictly linear (SL) receiver when the interfering users are (quasi-)synchronous with respect to the user of interest. High-powered interfering users, that may exist to support high data rates, increase the performance improvement delivered by the WL receiver. Moreover, it is shown that MAI can become proper, either identically or asymptotically, when users are asynchronous and equally powered. This is despite the fact that individual interfering signals are improper. Numerical results demonstrate that the WL receiver can outperform the SL receiver by 1-3 dB under the examined scenarios with current CDMA standards settings. In asynchronous or quasi-synchronous transmission modes, performance gain of the $W L$ receiver degrades unless the number of high-powered active users remains small. An example for implementation of the WL receiver is proposed and compared with that of the SL receiver when minimum-shift keying modulation, a special case of OQPSK, is used. The implementation is based on a fractionally spaced equalizer whose taps are updated by an adaptive algorithm. It is shown that the proposed structure is capable of delivering the maximum signal-to-noise ratio predicted by theory.
\end{abstract}

Index Terms-Bandlimited pulses, code-division multiple-access (CDMA), cyclostationarity (CS), fractionally spaced equalizer (FSE), improper noise, linear/conjugate-linear (L/CL) filtering, minimum-shift keying (MSK), offset quadrature phase-shift keying (OQPSK), widely linear (WL) receiver.

\section{INTRODUCTION}

$\mathbf{O}$ PTIMUM filtering of complex envelope signals requires replacing the classical strictly linear (SL) minimum mean-square error (MMSE) receiver with the linear/conju-

Paper approved by F. Santucci, the Editor for Transmission Systems of the IEEE Communications Society. Manuscript received December 8, 2003; revised August 19, 2004; April 13, 2005; and July 17, 2005. This paper was presented in part in at the IEEE Canadian Conference on Electrical and Computer Engineering, Niagara Falls, ON, Canada, May 2004.

A. Mirbagheri was with the Department of Electrical and Computer Engineering, University of Toronto, Toronto, ON M5S 3G4, Canada. He is currently with Qualcomm Inc., San Diego, CA 92121 USA (e-mail: arashm@qualcomm.com).

K. N. Plataniotis and S. Pasupathy are with the Department of Electrical and Computer Engineering, University of Toronto, Toronto, ON M5S 3G4, Canada (e-mail: kostas@dsp.utoronto.ca; pas@comm.utoronto.ca).

Digital Object Identifier 10.1109/TCOMM.2005.863766 gate-linear (L/CL) structure which processes both the received signal and its complex conjugate [1]. An alternative terminology for L/CL filtering is widely linear (WL) filtering, which was initiated in [2] and has prevailed over the last decade as the more popular. WL receivers find their application when the complementary autocorrelation (or pseudocovariance) functions of the complex envelope signals are nonzero, i.e., the signals are improper [3]. Improperness may originate from either the modulation scheme or coding method. A few examples of improper signal constellations are binary phase-shift keying (BPSK), offset quadrature phase-shift keying (OQPSK), minimum-shift keying (MSK), $M$-ary amplitude-shift keying (ASK), and simplex signals in general. The space-time block coding (STBC) scheme of [4] is also a coding example that results in improper signals.

This paper studies an enhanced WL receiver for OQPSKmodulated direct-sequence code-division multiple-access (DS-CDMA) systems that employ aperiodic pseudonoise (PN) sequences and bandlimited pulses. OQPSK (or staggered QPSK) modulation delays the quadrature signal component with respect to the in-phase component by a time offset equal to a fraction of a symbol period. Such delay limits the phase jumps to $\pm \pi / 2$ compared with $\pm \pi$ and $\pm \pi / 2$ in QPSK [5]. Current long-code CDMA standards (IS-95 and cdma2000) have adopted OQSPK modulation [6], [7].

Previous work on WL receivers for CDMA systems includes, but is not limited to, [8]-[16]. The importance of processing the complementary autocorrelation function in CDMA systems with improper multiple-access interference (MAI) was first demonstrated in [8] with BPSK modulation. In [9], the concept was further extended by deriving suboptimum and blind adaptive implementations. Performance analysis of the proposed receiver in [9] is presented in [10]. Blind adaptive WL receivers are also discussed in [11]-[13], where the authors arrive at implementations with different degrees of complexity. In [14], a low-complexity training-based adaptive WL receiver is discussed. WL equalization in the presence of the STBC scheme of [4] is the subject of [15]. Finally, in [16], the benefits of WL processing in frequency-selective fading channels are explored.

Attention to bandlimited pulses and cyclostationarity (CS) of the uncoded received signal are conspicuously absent in existing works on WL receivers for CDMA systems (including the aforementioned), and is the subject of this paper. Prior art has either explicitly assumed time-limited chip pulses (e.g., [8]-[12]) or considered discrete outputs of the chip-matched filter (CMF) with perfect carrier synchronization for all users 
(e.g., [13]-[16]). In this paper, focus is on the pre-CMF received signal, which is known to be a wide-sense cyclostationary (WSCS) random process [17]. By applying the frequency-shifting concept of [18] for bandlimited WSCS signals, new filters are designed that outperform the conventional CMF, known to maximize signal-to-noise ratio (SNR) only for Gaussian wide-sense stationary (WSS) signals. It will be shown that performance improvement of the WL receiver is directly related to the excess bandwidth of the chip pulse and chip delays of the interfering users.

Since the focus and approach of this work fundamentally differ from those in prior papers (e.g., [2] and [9]-[14]), the distinguishing features and their consequences on the receiver structure are clarified as the receiver formulation proceeds in Section III.

The contributions of this paper are as follows.

- The frequency response of the WL receiver for bandlimited pulses is derived and compared with that of the SL receiver. The extra components originating due to improper MAI are identified and analyzed. Expressions for SNR of both receivers are also presented and contrasted. A link between the concepts of (im)properness and CS is established. Moreover, a practical approach to the implementation of the WL receiver is also discussed. The implementation is based on an adaptive fractionally spaced equalizer (FSE) and is shown to deliver the theoretical maximum SNR. To disseminate the basic ideas and avoid notational complexity, this work proceeds in the additive white Gaussian noise (AWGN) channel. The design can be extended to frequency-selective channels, as was done in [19].

- Cases when interference becomes proper are identified. It is shown that for the ideal Nyquist chip pulse, interference turns to be a proper WSS random process and, consequently, the WL receiver reduces to the SL receiver. More interestingly, it is shown that if chip delays of the interfering users satisfy certain conditions, the pseudocovariance of MAI becomes identically zero, even though the individual interfering signals are improper. Users meeting these conditions are referred to as symmetric users. A large number of asynchronous users is shown to asymptotically form a symmetric set and make the MAI proper.

- Scenarios where the WL receiver delivers significant performance improvement over the SL receiver are discussed by contrasting the above cases. The WL receiver is shown to outperform the SL receiver when the interfering users are (quasi-)synchronous and high-powered with respect to the user of interest. This may arise in future generation systems that support high-data-rate services. Numerical results are presented to quantify the performance improvements.

The material presented in this paper applies to both OQPSK and MSK modulation schemes, as they only differ in the pulse shape. Numerical results, however, are more tailored toward the more spectrally efficient OQPSK with the square-root raisedcosine (Sqrt-RC) pulse.
The paper is organized as follows. Section II describes the system model. The design of the WL receiver is discussed in Section III. Section IV analyzes the performance of the WL receiver and compares it with that of the SL receiver. Section V presents an example for implementation of the receiver. Numerical results are presented in Section VI, with concluding remarks in Section VII.

\section{SySTEM MODEL}

Long-code DS-CDMA systems with OQPSK modulation is under review. This section describes the modeling of the received signal. The desired user is arbitrarily indexed with zero.

The received signal can be expressed as

$$
r(t)=\sum_{k=0}^{K} s_{k}(t)+\eta(t)
$$

using the complex baseband representation of passband signals. The second term $\eta(t)$ is a complex, circularly symmetric, zero-mean AWGN process with a two-sided noise power spectral density (PSD) of $\mathcal{N}_{0}$. The first term represents the sum of the received signals from each user, where $s_{k}(t)$ is the signal of user $k$. The baseband representation of $s_{k}(t)$ can be expressed as

$$
\begin{aligned}
s_{k}(t)= & \sqrt{\frac{P_{k}}{2}} e^{j \theta_{k}} \sum_{n=-\infty}^{\infty} b_{n}^{(\mathrm{I}, k)} a_{n}^{(\mathrm{I}, k)}\left(t-n T_{b}^{(k)}-\tau_{k}\right) \\
& -j b_{n}^{(\mathrm{Q}, k)} a_{n}^{(\mathrm{Q}, k)}\left(t-n T_{b}^{(k)}-\tau_{k}-\mathcal{T}\right)
\end{aligned}
$$

where the in-phase (I) and quadrature-phase (Q) signal components are appropriately notated. The symbols $P_{k}, \theta_{k}$, and $\tau_{k}$ represent, respectively, the signal power, phase offset, and arrival delay of user $k$. Code acquisition, carrier-phase and bit symbol timing synchronization for the desired user are assumed such that $\tau_{0}=0$ and $\theta_{0}=0$. The time offset corresponding to OQSPK modulation is constant for all users, and represented by $\mathcal{T} \in\left[0, T_{c}\right]$ where $1 / T_{c}$ is the system chip rate.

The spreading waveforms $a_{n}^{(\mathrm{I}, k)}(t)$ and $a_{n}^{(\mathrm{Q}, k)}(t)$, used to spread the information symbol $b_{n}^{(k)}=b_{n}^{(\mathrm{I}, k)}+j b_{n}^{(\mathrm{Q}, k)}$, are

$$
a_{n}^{(\mathcal{B}, k)}(t)=\sum_{i=0}^{N_{k}-1} a_{i+n N_{k}}^{(\mathcal{B}, k)} q\left(t-i T_{c}\right)
$$

where $a_{i}^{(\mathcal{B}, k)} \in\{ \pm 1\}$ represents the $i$ th chip of the PN sequence corresponding to the $\mathcal{B}$ branch of user $k$. The PN sequences are assumed to be equally likely independent and identically distributed (i.i.d.) random sequences with independence between the chips of different users. Mutual independence is also assumed among the bits and PN sequences of the I and Q branches. The spreading sequences associated with $b_{n}^{(\mathcal{B}, k)}$ are defined as $\boldsymbol{a}_{n}^{(\mathcal{B}, k)}=\left[a_{n N_{k}}^{(\mathcal{B}, k)}, a_{n N_{k}+1}^{(\mathcal{B}, k)}, \ldots, a_{n N_{k}+N_{k}-1}^{(\mathcal{B}, k)}\right]$. The ratio of the bit period of user $k$ to the chip period, $N_{k}=T_{b}^{(k)} / T_{c}$, is referred to as the spreading factor of that user. The chip pulse $q(t)$ is assumed to be real and bandlimited, either accurately or approximately, with an excess bandwidth of $\alpha$. For instance, the Sqrt-RC pulse with $\alpha$ excess bandwidth is strictly limited to $|f| \leq(1+\alpha) /\left(2 T_{c}\right)$. The half-cosine pulse, on the other hand, 
can be truncated in the frequency domain to approximately represent a bandlimited pulse, where its excess bandwidth $\alpha$ depends on the desired level of accuracy. The processing gain (or bandwidth-expansion factor) of user $k$ is $N_{k}(1+\alpha)$.

The above setting covers most radio configurations (RC) defined in IS-95 and cdma2000 standards [6], [7]. For instance, binary information bits $\left(b_{n}^{(\mathrm{I}, k)}=b_{n}^{(\mathrm{Q}, k)}\right)$ with OQPSK spreading $\left(\mathcal{T}=0.5 T_{c}\right)$ results in reverse fundamental and supplemental channel structure of RC1 and RC2. Alternatively, quaternary information bits $\left(b_{n}^{(\mathrm{I}, k)} \neq b_{n}^{(\mathrm{Q}, k)}\right)$ with QPSK spreading $(\mathcal{T}=0)$ results in a reverse traffic channel structure of RC3 and RC4. Furthermore, the OQPSK signals become MSK signals with $\mathcal{T}=T_{c}$ and a half-cosine pulse as the chip pulse [20].

The system model also supports multirate CDMA since, in general, $N_{k} \neq N_{k^{\prime}}$ for $k \neq k^{\prime}$, leading to multiple bit rates for different users. Moreover, if the baseband signal defined in (2) experiences a delay of $\tau_{k}$, it can alternatively be expressed as

$$
\begin{array}{r}
s_{k}(t)=\sqrt{\frac{P_{k}}{2}} e^{j \theta_{k}} \sum_{n=-\infty}^{\infty} d_{n}^{(\mathrm{I}, k)} q\left(t-T_{k}-n T_{c}\right) \\
-j d_{n}^{(\mathrm{Q}, k)} q\left(t-T_{k}-n T_{c}-\mathcal{T}\right)
\end{array}
$$

where

$$
d_{n}^{(\mathcal{B}, k)}=b_{\left\lfloor\left(n-J_{k}\right) / N_{k}\right\rfloor}^{(\mathcal{B}, k)} a_{n-J_{k}}^{(\mathcal{B}, k)}
$$

$J_{k}=\left\lfloor\tau_{k} / T_{c}\right\rfloor$, and $T_{k}=\bmod \left(\tau_{k}, T_{c}\right)$ represent, respectively, the direct-sequence spread bits, the bit delay as an integer multiple of $T_{c}$, and the bit delay modulo the chip period such that $T_{k} \in\left[0, T_{c}\right)$ and $\tau_{k}=J_{k} T_{c}+T_{k}$. The floor function $\lfloor x\rfloor$ returns the integer portion of a real number $x$. The term $T_{k}$ is referred to as the chip delay of the $k$ th user. With such reformulation, from the perspective of user $0, d_{n}^{(\mathcal{B}, k)} \in\{ \pm 1\}$ can be effectively modeled as equally likely i.i.d. random sequences. Thus, the effects of the interferer bits and integer bit delays disappear under the random spreading assumption.

\section{WIDELY LINEAR RECEIVER}

This section treats the design of the WL receiver. The design approach employs the same tools used in [19] and [21]. The distinguishing feature, however, is the presence of improper complex noise, which results in two distinct integral equations to be solved. The integral equations are first presented, followed by their solutions and a discussion on the structure of the WL receiver.

\section{A. Integral Equations}

For optimum filtering of complex envelope signals, the conventional SL receiver structure must be replaced by the $\mathrm{L} / \mathrm{CL}$ structure of Fig. 1 [22]. Both the received signal and its conjugate are linearly processed by two time-variant filters $\phi_{n}(t)$ and $\psi_{n}(t)$, where the index $n$ denotes the filter responses associated with the detection of $b_{n}^{(0)}$. The conjugate linear branch in Fig. 1 is absent in the classical linear receiver, and bears significance

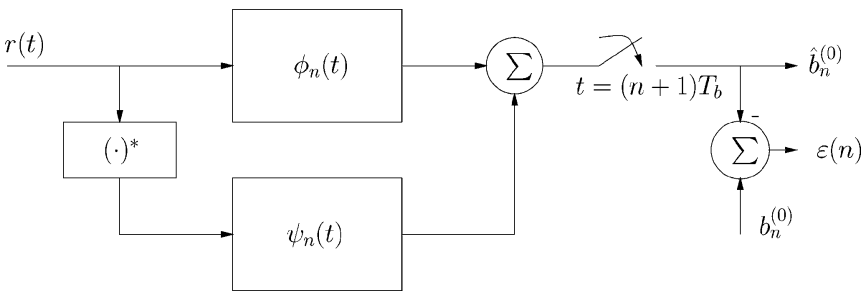

Fig. 1. L/CL filtering structure for user 0 .

for improper signals. Let $N=N_{0}$ and $T_{b}=T_{b}^{(0)}$. The estimation error is $\varepsilon(n)=b_{n}^{(0)}-\hat{b}_{n}^{(0)}$, where $\hat{b}_{n}^{(0)}$ can be expressed as

$$
\hat{b}_{n}^{(0)}=\int_{-\infty}^{\infty} r(u) \phi_{n}\left(T_{b}-u\right)+r^{*}(u) \psi_{n}\left(T_{b}-u\right) d u .
$$

The two filters, $\phi_{n}(t)$ and $\psi_{n}(t)$, are designed to minimize the mean-square error $\mathcal{J}_{n}=\mathrm{E}\left[|\varepsilon(n)|^{2}\right]$, where $\mathrm{E}[\cdot]$ represents the expectation operation. They are determined using the two orthogonality conditions for complex envelopes [22]: $\mathrm{E}[\varepsilon(n) r(t)]=0$ and $\mathrm{E}\left[\varepsilon(n) r^{*}(t)\right]=0$. Substituting $\varepsilon(n)$ and $\hat{b}_{n}^{(0)}$ into the orthogonality conditions yields the following two Fredholm integral equations of the first kind:

$$
\begin{gathered}
\int_{-\infty}^{\infty} R_{r r}(t, u) \phi_{n}\left(T_{b}-u\right)+R_{r r^{*}}(t, u) \psi_{n}\left(T_{b}-u\right) d u \\
\quad=\mathrm{E}\left[b_{n}^{(0)} r(t)\right] \\
\int_{-\infty}^{\infty} R_{r r^{*}}^{*}(t, u) \phi_{n}\left(T_{b}-u\right)+R_{r r}^{*}(t, u) \psi_{n}\left(T_{b}-u\right) d u \\
\quad=\mathrm{E}\left[b_{n}^{(0)} r^{*}(t)\right]
\end{gathered}
$$

for $-\infty<t<\infty$, where $R_{x x^{*}}(t, u)=E\left[x(t) x^{*}(u)\right]$ and $R_{x x}(t, u)=E[x(t) x(u)]$ denote, respectively, the autocorrelation function and complementary autocorrelation function of a complex random process $x(t)$ [5, p. 312]. The expectation operations are conditioned on the knowledge of PN sequences of the desired user plus the signal powers, arrival delays, and phase offsets of all users.

The right-hand sides (RHS) of (6) are forcing functions of the integral equations, i.e., the received signal associated with $b_{n}^{(0)}$. By moving the desired signal components of $r(t)$ to the RHS, (6) can alternatively be expressed as

$$
\begin{gathered}
\int_{-\infty}^{\infty} R_{n n}(t, u) \phi_{n}\left(T_{b}-u\right)+R_{n n^{*}}(t, u) \psi_{n}\left(T_{b}-u\right) d u \\
=\alpha_{n} a_{n}^{(\mathrm{I}, 0)}\left(t-n T_{b}\right)+\beta_{n} a_{n}^{(\mathrm{Q}, 0)}\left(t-n T_{b}-\mathcal{T}\right) \\
\int_{-\infty}^{\infty} R_{n n^{*}}^{*}(t, u) \phi_{n}\left(T_{b}-u\right)+R_{n n}^{*}(t, u) \psi_{n}\left(T_{b}-u\right) d u \\
=\alpha_{n} a_{n}^{(\mathrm{I}, 0)}\left(t-n T_{b}\right)-\beta_{n} a_{n}^{(\mathrm{Q}, 0)}\left(t-n T_{b}-\mathcal{T}\right)
\end{gathered}
$$

where $R_{n n^{*}}(t, u)$ and $R_{n n}(t, u)$ are now the autocorrelation and complementary autocorrelation functions of noise only, defined as

$$
n(t)=\sum_{k=1}^{K} s_{k}(t)+\eta(t) .
$$


The scalars $\alpha_{n}$ and $\beta_{n}$ absorb the desired signal components from the left-hand sides and are defined as

$$
\begin{aligned}
\alpha_{n}= & \sqrt{\frac{P_{0}}{2}}-\frac{P_{0}}{2} \int_{-\infty}^{\infty} a_{n}^{(\mathrm{I}, 0)}\left(u-n T_{b}\right) \phi_{n}\left(T_{b}-u\right) \\
& +a_{n}^{(\mathrm{I}, 0)}\left(u-n T_{b}\right) \psi_{n}\left(T_{b}-u\right) d u \\
\beta_{n}= & \sqrt{\frac{P_{0}}{2}}+\frac{P_{0}}{2} \int_{-\infty}^{\infty} a_{n}^{(\mathrm{Q}, 0)}\left(u-n T_{b}-\mathcal{T}\right) \phi_{n}\left(T_{b}-u\right) \\
& -a_{n}^{(\mathrm{Q}, 0)}\left(u-n T_{b}-\mathcal{T}\right) \psi_{n}\left(T_{b}-u\right) d u .
\end{aligned}
$$

It is shown in Appendix I that $\alpha_{n}$ and $\beta_{n}$ are directly related to $\mathrm{SNR}$, and $\alpha_{n}=\beta_{n}$. Defining $\phi_{n}(t)$ as $\phi_{n}(t)=\alpha_{n} g_{n}(t)$ and $\psi_{n}(t)$ as $\psi_{n}(t)=\alpha_{n} h_{n}(t)$, the equations of (7) can be further simplified to

$$
\begin{gathered}
\int_{-\infty}^{\infty} R_{n n}(t, u) g_{n}\left(T_{b}-u\right)+R_{n n^{*}}(t, u) h_{n}\left(T_{b}-u\right) d u \\
=a_{n}^{(\mathrm{I}, 0)}\left(t-n T_{b}\right)+a_{n}^{(\mathrm{Q}, 0)}\left(t-n T_{b}-\mathcal{T}\right) \\
\int_{-\infty}^{\infty} R_{n n^{*}}^{*}(t, u) g_{n}\left(T_{b}-u\right)+R_{n n}^{*}(t, u) h_{n}\left(T_{b}-u\right) d u \\
=a_{n}^{(\mathrm{I}, 0)}\left(t-n T_{b}\right)-a_{n}^{(\mathrm{Q}, 0)}\left(t-n T_{b}-\mathcal{T}\right)
\end{gathered}
$$

where (11a)-(11b) appear at the bottom of the page.

It can be observed that, in general, the complementary autocorrelation function of (11a) is nonzero for $\mathcal{T} \neq 0$. Therefore, the OQPSK modulation introduces improper complex noise into the system. However, phase locking on all the interfering users is necessary [1], otherwise, it is easy to show that $R_{n n}(t, u)=0$ for i.i.d. phase offsets with uniform distribution $\theta_{k} \in U[0,2 \pi)$. Henceforth, it is assumed that the receiver locks on phase offsets $\theta_{k}$ and compensates for them. As the knowledge of phase offsets becomes available, compensating for them turns out to be straightforward. Phase estimation can be achieved with the aid of pilot signals that are already incorporated in CDMA standards [6], [7]. After phase estimation and compensation, $R_{n n}(t, u)$ can be rewritten with $\theta_{k}=0$ for all $k$. It is also noted that the noise considered in (11) consists of MAI and AWGN only. Intersymbol interference (ISI) is neglected. This is justified, as chip pulses considered either satisfy the Nyquist-I criterion (e.g., the Sqrt-RC pulse) or are time-limited to $|t| \leq 2 T_{c}$ (e.g., the half-cosine pulse) in the ISI-free AWGN channel. Next, the solutions to the filters $g_{n}(t)$ and $h_{n}(t)$ are presented.

\section{B. Solutions}

It is straightforward to show that the noise defined by (8) and (11) has zero mean and is periodic in the second moment. Hence, it is a WSCS random process with a period of $T_{\mathrm{CS}}=T_{c}$ [17], [23]. As in [19] and [21], the harmonic series representation (HSR) technique [22] is used to solve for $g_{n}(t)$ and $h_{n}(t)$.
The HSR technique accomplishes a decomposition of a WSCS process into a countable set of jointly WSS and bandlimited processes. Hence, a scalar WSCS process can be treated as a WSS vector process. In such case, the integral equations of (10) reduce to convolution integrals, and can alternatively be expressed in the frequency domain by the following matrix equations:

$$
\begin{aligned}
\mathbb{S} \mathbb{G}_{n}+\mathbb{R} \mathbb{H}_{n} & =\mathbb{Q}_{n}^{+} \\
\mathbb{R}^{*} \mathbb{G}_{n}+\mathbb{S}^{*} \mathbb{H}_{n} & =\mathbb{Q}_{n}^{-}
\end{aligned}
$$

where $\mathbb{G}_{n}$ and $\mathbb{H}_{n}$ are vectors containing harmonics of the $g_{n}(t)$ and $h_{n}(t)$, respectively. Also, $\mathbb{Q}_{n}^{+}$and $\mathbb{Q}_{n}^{-}$are vectors containing the forcing functions of (10a) and (10b). The matrices corresponding to autocorrelation and complementary autocorrelation functions are represented by $\mathbb{R}$ and $\mathbb{S}$. The detailed mathematical description of all the above elements can be found in Appendix II.

The matrix equations of (12) appear to be similar to those in [2]. However, their formations are fundamentally different. The matrices involved in [2] (and [9]-[16]) are determined by the observation vector which constitutes samples of the filtered received signal in the time domain. In contrast, the vectors and matrices in (12) are determined by the HSR of the filters and received signal spectrum. Whereas the dimensions of $\mathbb{R}$ and $\mathbb{S}$ correspond to the number of observation samples in [2] and [9]-[16], here they are determined by $\alpha$, the excess bandwidth of the pulse shape in (12).

Prior to presenting the solutions to $\mathbb{G}_{n}$ and $\mathbb{H}_{n}$, some interesting properties of $\mathbb{R}$ and $\mathbb{S}$ are reviewed. From Appendix II, it can be shown that $\mathbb{R}$ is Hermitian $\left(\mathbb{R}^{H}=\mathbb{R}\right)$ and nonsingular. However, $\mathbb{S}$ is always singular, and $\mathbb{S}^{\prime}=\mathbb{S}$. Also, the arrays on the longest diagonal of $\mathbb{S}$ perpendicular to its main diagonal are zero.

Accounting for the singularity of $\mathbb{S}$, the solutions to the unknown filters are

$$
\begin{aligned}
\mathbb{G}_{n}= & {\left[\mathcal{A}_{n}^{(\mathrm{I}, 0)}\left(e^{j 2 \pi f T_{c}}\right)+\mathcal{A}_{n}^{(\mathrm{Q}, 0)}\left(e^{j 2 \pi f T_{c}}\right)\right] e^{-j 2 \pi f T_{b}} \mathbb{G}_{1} } \\
& +\left[\mathcal{A}_{n}^{(\mathrm{I}, 0)}\left(e^{j 2 \pi f T_{c}}\right)-\mathcal{A}_{n}^{(\mathrm{Q}, 0)}\left(e^{j 2 \pi f T_{c}}\right)\right] e^{-j 2 \pi f T_{b}} \mathbb{G}_{2}
\end{aligned}
$$

where $\mathbb{G}_{1}$ and $\mathbb{G}_{2}$ are time-invariant filters defined as

$$
\begin{aligned}
& \mathbb{G}_{1}=\left(\mathbb{S}^{*} \mathbb{R}^{-1} \mathbb{S}-\mathbb{R}^{*}\right)^{-1} \mathbb{S}^{*} \mathbb{R}^{-1} \mathbb{Q} \mathbb{U} \\
& \mathbb{G}_{2}=\left(\mathbb{R}^{*}-\mathbb{S}^{*} \mathbb{R}^{-1} \mathbb{S}\right)^{-1} \mathbb{Q} \mathbb{U} .
\end{aligned}
$$

Similarly

$$
\begin{aligned}
& \mathbb{H}_{n}=\left[\mathcal{A}_{n}^{(\mathrm{I}, 0)}\left(e^{j 2 \pi f T_{c}}\right)+\mathcal{A}_{n}^{(\mathrm{Q}, 0)}\left(e^{j 2 \pi f T_{c}}\right)\right] e^{-j 2 \pi f T_{b}} \mathbb{H}_{1} \\
& +\left[\mathcal{A}_{n}^{(\mathrm{I}, 0)}\left(e^{j 2 \pi f T_{c}}\right)-\mathcal{A}_{n}^{(\mathrm{Q}, 0)}\left(e^{j 2 \pi f T_{c}}\right)\right] e^{-j 2 \pi f T_{b}} \mathbb{H}_{2}
\end{aligned}
$$

$$
\begin{aligned}
R_{n n}(t, u) & =\sum_{k=1}^{K} \sum_{n=-\infty}^{\infty} \frac{P_{k}}{2} e^{j 2 \theta_{k}}\left[q\left(t-n T_{c}-\tau_{k}\right) q\left(u-n T_{c}-\tau_{k}\right)-q\left(t-n T_{c}-\tau_{k}-\mathcal{T}\right) q\left(u-n T_{c}-\tau_{k}-\mathcal{T}\right)\right] \\
R_{n n^{*}}(t, u) & =\sum_{k=1}^{K} \sum_{n=-\infty}^{\infty} \frac{P_{k}}{2}\left[q\left(t-n T_{c}-\tau_{k}\right) q\left(u-n T_{c}-\tau_{k}\right)+q\left(t-n T_{c}-\tau_{k}-\mathcal{T}\right) q\left(u-n T_{c}-\tau_{k}-\mathcal{T}\right)\right]+\mathcal{N}_{0}
\end{aligned}
$$




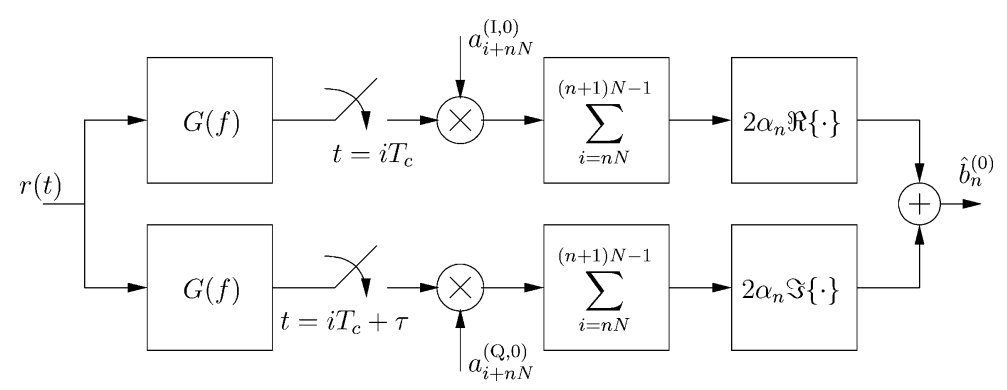

Fig. 2. WL receiver structure for user 0 .

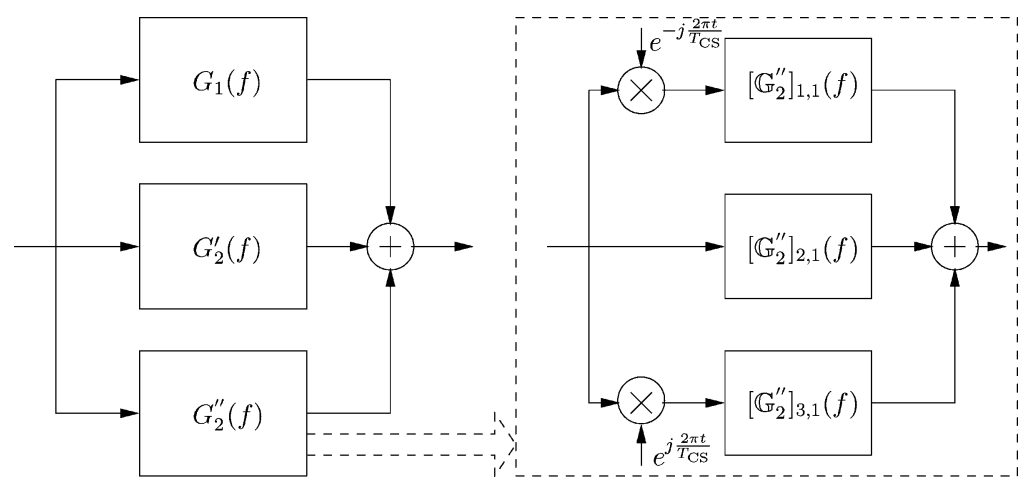

Fig. 3. Broken-down structure of $G(f)$ with the dashed block zoomed in on $\mathbb{G}^{\prime \prime}(f)$ to illustrate the frequency shifters and filters.

where $\mathbb{H} 1$ and $\mathbb{H}_{2}$ are

$$
\mathbb{H}_{1}=\mathbb{G}_{2}^{*} \quad \mathbb{H}_{2}=\mathbb{G}_{1}^{*} .
$$

As expected from [22], the solutions to $\mathbb{G}_{n}$ and $\mathbb{H}_{n}$ are comprised of two parts: time-variant components consisting of the $Z$-transforms of the PN sequences responsible for despreading the desired signal, and time-invariant components $\mathbb{G}_{1}$ and $\mathbb{G}_{2}$ which replace the CMF. The exponential component $e^{-j 2 \pi f T_{b}}$ in (14) and (16) simply make the filters causal. It is noted that the $Z$-transforms and the exponential component are scalars and invariant to frequency shifts introduced by HSR. It is also worth mentioning that the solutions of $\mathbb{G}_{1}$ and $\mathbb{G}_{2}$ involve the inverse of Schur complement of $\mathbb{R}$ with respect to the augmented covariance matrix, as defined in [24]. ${ }^{1}$

\section{Receiver Structure}

The WL receiver accounts for the complementary autocorrelation functions which were overlooked in the design of the SL receiver. It can be easily verified that setting $\mathbb{S}=0$ results in $\mathbb{G}_{1}=0$ and $\mathbb{G}_{2}=\mathbb{R}^{*-1} \mathbb{Q U}$, which is identical to the solution of the SL receiver formulated in [21]. The extra components resulting from $\mathbb{S} \neq 0$ in the WL receiver structure can also be illustrated via the following reformulation of $\mathbb{G}_{2}$ :

$$
\begin{aligned}
& \mathbb{G}_{2}=\left[\mathbb{R}^{*}(\mathbb{\square}-\mathbb{A})\right]^{-1} \mathbb{Q U} \\
& =(\mathbb{\mathbb { A }}-\mathbb{A})^{-1} \mathbb{R}^{*-1} \mathbb{Q U} \\
& =\underbrace{\mathbb{R}^{*-1} \mathbb{Q U}}_{\mathbb{G}_{2}^{\prime \prime}}+\underbrace{A \mathbb{R} \mathbb{R}^{*-1} \mathbb{Q U}+A^{2} \mathbb{R}^{*-1} \mathbb{Q U}+\cdots}_{\mathbb{G}_{2}^{\prime}}
\end{aligned}
$$

\footnotetext{
${ }^{1}$ The Schur complement of $\mathbb{R}$ with respect to the augmented covariance matrix of $\left(\begin{array}{cc}\mathbb{R} & \mathbb{S} \\ \mathbb{S}^{*} & \mathbb{R}^{*}\end{array}\right)$ is $\mathbb{R}^{*}-\mathbb{S}^{*} \mathbb{R}^{-1} \mathbb{S}$.
}

where $A=\mathbb{R}^{*-1} \mathbb{S}^{*} \mathbb{R}^{-1} \mathbb{S}$ and the last line of (17) is obtained from the matrix series expansion

$$
(\mathbb{\square}-\mathbb{A})^{-1}=\mathbb{\square}+A+A^{2}+\cdots
$$

for an arbitrary square matrix $A$. In (17), the vector $\mathbb{G}_{2}^{\prime \prime}$ is the solution to the SL receiver, and the vector $\mathbb{G}_{2}^{\prime}$ arises due to improper complex noise.

The structure of the WL receiver can be shown to simplify to that in Fig. 2 after accounting for equations in (16). The new filter, $G(f)$, whose broken-down structure is shown in Fig. 3, replaces the CMF and is expressed as $G(f)=G_{1}(f)+G_{2}^{\prime}(f)+$ $G_{2}^{\prime \prime}(f)$ where $G_{1}(f), G_{2}^{\prime}(f)$, and $G_{2}^{\prime \prime}(f)$ are constructed from a parallel bank of frequency shifters, each followed by their corresponding filters formulated in $\mathbb{G}_{1}, \mathbb{G}_{2}^{\prime}$, and $\mathbb{G}_{2}^{\prime \prime}$, respectively. This is illustrated for $\mathbb{G}_{2}^{\prime \prime}$ in the dashed block of Fig. 3 [18]. The top and bottom branches in Fig. 2 estimate $b_{n}^{(\mathrm{I}, 0)}$ and $b_{n}^{(\mathrm{Q}, 0)}$, respectively, with $\Re\{\cdot\}$ and $\Im\{\cdot\}$ representing the real and imaginary operators.

\section{Performance Analysis}

This section analyzes the WL receiver. Special cases when the WL receiver reduces to the SL receiver, either identically $(\mathbb{S}=$ 0 ) or asymptotically ( $\mathbb{S} \rightarrow 0$ ), will be investigated. Also, scenarios when the WL receiver delivers maximum performance improvement over the SL receiver will be discussed. The SNR expressions of both receivers will be presented and compared in the end.

A. $\mathbb{S}=0$

Of interest is the study of cases when $\mathbb{S}=0$. To identify such cases, the expression for $\mathbb{S}$ is rewritten as $\mathbb{S}=\sum_{k=1}^{K} \mathbb{S}_{k}$, 
where $\mathbb{S}_{k}$ is the complementary autocorrelation function corresponding to user $k$, defined similar to (40), except for replacing $\mathbb{P}^{\mathbb{S}}$ with $\mathbb{P}^{\mathbb{S}_{k}}$, as defined in (19), shown at the bottom of the page.

In the above, $T_{\mathrm{CS}}=T_{c}$. This matrix representation is valid for $\alpha<200 \%$, but can easily be extended to larger values of excess bandwidth.

Four cases can occur that yield proper noise: 1) $\mathcal{T}=0$; ) $K=0$; 3) $\alpha=0$; and 4) symmetric interferers. The first case yields balanced QPSK where the WL receiver simplifies to the SL receiver. In the second and third cases, the noise becomes a WSS random process with the new filters reducing to the CMF. Moreover, all matrices and vectors become scalars for $\alpha=0$. The last case requires a closer look at $\mathbb{P}^{\mathbb{S}_{k}}$, and is now investigated.

Symmetric interferers are hereby defined as a set of interfering users whose collective sum of nonzero complementary autocorrelation functions $\left(\forall_{k} \mathbb{S}_{k} \neq 0\right)$ is zero. A system has symmetric interferers, if for every distinct interferer ${ }^{2}$ with power $P_{k}$ and chip delay $T_{k}$, there exists two other interferers with equal power and chip delays of $\bmod \left(T_{k}+T_{\mathrm{CS}} / 2, T_{\mathrm{CS}}\right)$ and $\bmod \left(T_{k}+T_{\mathrm{CS}} / 4, T_{\mathrm{CS}}\right)$. More precisely

$$
\begin{aligned}
& \forall\left\{P_{k}, T_{k}\right\}, \exists\left\{P_{k^{\prime}}=P_{k}, T_{k^{\prime}}=\bmod \left(T_{k}+\frac{T_{\mathrm{CS}}}{2}, T_{\mathrm{CS}}\right)\right\} \\
& \forall\left\{P_{k}, T_{k}\right\}, \exists\left\{P_{k^{\prime \prime}}=P_{k}, T_{k^{\prime \prime}}=\bmod \left(T_{k}+\frac{T_{\mathrm{CS}}}{4}, T_{\mathrm{CS}}\right)\right\} .
\end{aligned}
$$

If these two conditions are met, it can be shown that $\mathbb{S}=$ 0 by examining the arrays of (19). For instance, with $T_{\mathrm{CS}}=$ $T_{c}$ and $K=4$ equally powered users with chip delays $\boldsymbol{T}=$ $[0.15,0.40,0.65,0.90] T_{c}$ create a set of symmetric interferers. If $\mathcal{T}=0.5 T_{c}$, as with the case in CDMA standards [6], [7], the main diagonal of $\mathbb{S}_{k}$ is also zero and, consequently, only (20a) needs to be satisfied to make the interferers symmetric.

\section{B. $\mathbb{S} \rightarrow 0$}

In addition to the previous four cases where $\mathbb{S}$ turned out to be identically zero, another scenario can occur that makes $\mathbb{S} \rightarrow 0$. The nonzero arrays of $\mathbb{P}^{\mathbb{S}_{k}}$ in (19) are all comprised of an $e^{-j p \pi T_{k} / T_{c}}$ term $(p=2,4)$ whose expectation is zero

\footnotetext{
${ }^{2}$ As evident from the system model, the average impact of two users with identical chip delays is the same in long-code CDMA systems. Two users are therefore referred to as distinct users if their chip delays differ.
}

for $T_{k} \in U\left[0, T_{c}\right)$. As the number of equally powered asynchronous interfering users with i.i.d. chip delays of such distribution grows in the system, the sum $\sum_{k=1}^{K} \mathbb{P}^{\mathbb{S}_{k}}$ asymptotically approaches the statistical average of $\mathbb{P}^{S_{k}}$

$$
\lim _{K \rightarrow \infty} \sum_{k=1}^{K} \mathbb{P}^{\mathbb{S}_{k}}=\mathrm{E}\left[\mathbb{P}^{\mathbb{S}_{k}}\right]=0 .
$$

This can also be intuitively explained. As the number of active users in the system with uniform power distribution increases, the probability of forming a symmetric or near-symmetric set of interferers grows, and the effect of $\mathbb{S}$ gradually diminishes. Simulations demonstrate that $K=30$ is large enough to make $\mathbb{S} \rightarrow 0$.

\section{C. $\mathbb{S} \gg 0$}

Naturally, scenarios where the WL receiver outperforms the SL receiver can be identified by counterarguing the cases discussed so far. The symmetry that results in $\mathbb{S}=0$ or $\mathbb{S} \rightarrow 0$ is violated when interfering users demonstrate one or both of the conditions of nonuniform power distribution and (quasi-)synchronism. Nonuniform power distribution may occur in nextgeneration CDMA systems which support various data rates with different qualities of service (QoS). Synchronism between users takes place on the forward link, where the base station transmits the signals of all users simultaneously. In the reverse link, however, only quasi-synchronism can be achieved with the aid of a timing reference. Quasi-synchronous CDMA signals can be modeled with chip delays chosen from a probability distribution function $f_{T}(\cdot)$, which is nonzero in $\left[0, \Delta_{m}\right]$ for $0<$ $\Delta_{m}<T_{c}$ [25]. For instance, in wideband CDMA (WCDMA) systems, all users are synchronized within $\Delta_{m}=T_{c} / 8$ in the reverse link to reduce intracell interference [26]. It will be shown in the next section that even quasi-synchronous signals yield remarkable gains in favor of the WL receiver, as the tightened range of allowed chip delays prevents forming symmetric interferers.

\section{Signal-to-Noise Ratio}

The expressions for SNR of the WL and SL receivers are now presented. Following Appendix I and the steps in [8], the complex baseband SNR expression for the WL receiver in the I phase after the real operator can be written as in (22), shown at the bottom of the page, where

$$
\begin{aligned}
\xi(t, u)= & \int_{-\infty}^{\infty}\left[R_{n n}(t, u) g_{n}\left(T_{b}-u\right)+R_{n n^{*}}(t, u) h_{n}\left(T_{b}-u\right)\right. \\
& \left.+R_{n n^{*}}^{*}(t, u) g_{n}\left(T_{b}-u\right)+R_{n n}^{*}(t, u) h_{n}\left(T_{b}-u\right)\right] d u .
\end{aligned}
$$

$$
\mathbb{P}^{\mathbb{S}_{k}}=\frac{P_{k}}{2 T_{c}}\left[\begin{array}{ccc}
e^{j 4 \pi T_{k} / T_{c}}\left(1-e^{j 4 \pi \mathcal{T} / T_{c}}\right) & e^{j 2 \pi T_{k} / T_{c}}\left(1-e^{j 2 \pi \mathcal{T} / T_{c}}\right) & 0 \\
e^{j 2 \pi T_{k} / T_{c}}\left(1-e^{j 2 \pi \mathcal{T} / T_{c}}\right) & 0 & e^{-j 2 \pi T_{k} / T_{c}}\left(1-e^{-j 2 \pi \mathcal{T} / T_{c}}\right) \\
0 & e^{-j 2 \pi T_{k} / T_{c}}\left(1-e^{-j 2 \pi \mathcal{T} / T_{c}}\right) & e^{-j 4 \pi T_{k} / T_{c}}\left(1-e^{-j 4 \pi \mathcal{T} / T_{c}}\right)
\end{array}\right]
$$

$$
\mathrm{SNR}_{n}^{\mathrm{WL}, \mathrm{I}}=\frac{\frac{P_{0}}{2}\left\{\int_{-\infty}^{\infty} \Re\left[a_{n}^{(\mathrm{I}, 0)}\left(u-n T_{b}\right) g_{n}\left(T_{b}-u\right)+a_{n}^{(\mathrm{I}, 0)}\left(u-n T_{b}\right) h_{n}\left(T_{b}-u\right)\right] d u\right\}^{2}}{\Re\left\{\frac{1}{2} \int_{-\infty}^{\infty} g_{n}\left(T_{b}-u\right) \xi(t, u)+h_{n}\left(T_{b}-u\right) \xi(t, u) d u\right\}}
$$




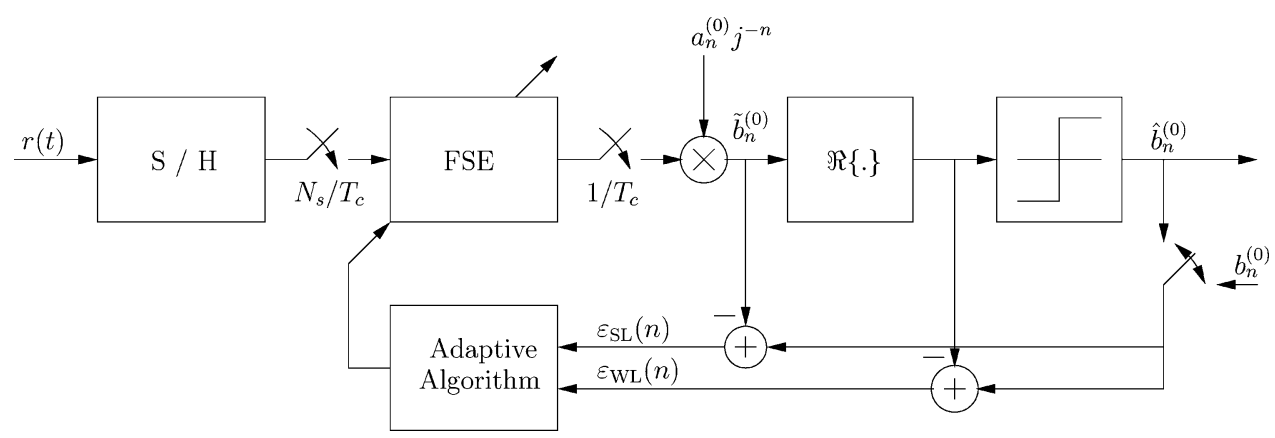

Fig. 4. Adaptive receiver architecture with an updating period of $T_{c}$.

If the integral equations in (10) hold, then (22) can be further simplified to

$$
\begin{aligned}
\mathrm{SNR}_{n}^{\mathrm{WL}, \mathrm{I}}= & \frac{P_{0}}{2} \int_{-\infty}^{\infty} \Re\left[a_{n}^{(\mathrm{I}, 0)}\left(u-n T_{b}\right) g_{n}\left(T_{b}-u\right)\right. \\
& \left.+a_{n}^{(\mathrm{I}, 0)}\left(u-n T_{b}\right) h_{n}\left(T_{b}-u\right)\right] d u .
\end{aligned}
$$

As shown in Appendix I, $\mathrm{SNR}_{n}^{\mathrm{WL}}=2 \mathrm{SNR}_{n}^{\mathrm{WL}, \mathrm{I}}$. An alternative, and yet computationally efficient, form can be expressed in the frequency domain by using the inverse Fourier representation of signals and their HSR as

$$
\mathrm{SNR}^{\mathrm{WL}}=2 N P_{0} \Re\left\{\int_{-1 / 2 T_{c}}^{1 / 2 T_{c}} \cup \mathbb{Q}\left(\mathbb{G}_{1}+\mathbb{G}_{2}\right) d f\right\}
$$

where the effect of PN spreading sequences are averaged out. Similarly, the SNR expression for the SL receiver is

$$
\mathrm{SNR}^{\mathrm{SL}}=2 N P_{0} \Re\left\{\int_{-1 / 2 T_{c}}^{1 / 2 T_{c}} \mathbb{U} \mathbb{Q}\left(\mathbb{G}_{2 \mid \mathbb{S}=0}\right) d f\right\}
$$

where the SL receiver is evaluated by setting $\mathbb{S}=0$ in $\mathbb{G}_{2}$. The relative performance improvement of the WL receiver over the $S L$ receiver, the parameter of interest in this paper, can therefore be formulated as

$$
\Delta \mathrm{SNR}=\frac{\Re\left\{\int_{-1 / 2 T_{c}}^{1 / 2 T_{c}} \mathbb{U} \mathbb{Q}\left(\mathbb{G}_{1}+\mathbb{G}_{2}\right) d f\right\}}{\Re\left\{\int_{-1 / 2 T_{c}}^{1 / 2 T_{c}} \cup \mathbb{Q}\left(\mathbb{G}_{2 \mid \mathbb{S}=0}\right) d f\right\}} .
$$

Numerical results on performance comparison of the two receivers are based on evaluating $\triangle \mathrm{SNR}$ for different scenarios.

It is reiterated that the design, performance analysis, and formulation of SNR expressions presented here apply to any pulse shape, as long as its frequency response has even symmetry. Hence, the preceding discussions cover both OQPSK and MSK modulation schemes.

\section{RECEIVER IMPLEMENTATION}

In the previous two sections, the SNR-maximizing WL receiver was accurately and explicitly derived and analyzed. To avoid any form of approximation or compromise in performance, the continuous-time impulse response of the filter was not restricted to a finite time support. To address this impractical assumption, an example of receiver implementation via adaptive algorithms is discussed here. The proposed architecture operates in discrete time, and is based on a finite impulse response (FIR) FSE.

\section{A. Architecture}

Fig. 4 depicts the proposed structure of the adaptive receiver for user 0. The FSE, whose structure can be found in [19], is the basic block of the structure. In [27], it is shown that exploiting the CS of the received signal through frequency shifting, as illustrated in the dashed block of Fig. 3, can also be realized by FSEs.

For simplicity, the adaptive receiver is based on binary MSK, which is a simplified form of the OQPSK model as described in Section II. With binary information bits and PN sequences, the notions of I and Q are dropped in this section.

The received signal is processed by a "Sample and Hold" (S/H) block, which is assumed to be ideal with impulse response $h_{\mathrm{S} / \mathrm{H}}(t)=\delta(t)$. The $\mathrm{S} / \mathrm{H}$ output is oversampled at the rate of $T_{s}=T_{c} / N_{s}$, where $N_{s}>1$. The samples are next input to the FSE and stacked in a $(M+1) \times 1$ vector of $\boldsymbol{x}(n)$ defined as

$\boldsymbol{x}(n)=\left[r\left(n T_{c}-T_{D}\right), r\left(n T_{c}-T_{D}+T_{s}\right), \ldots, r\left(n T_{c}-T_{D}+M T_{s}\right)\right]^{\prime}$

where $T_{D}$ is a delay to make the receiver causal and center $d_{n}^{(0)}=b_{\lfloor n / N\rfloor}^{(0)} a_{n}^{(0)}$ in the FSE. The FSE has $M+1$ complex tap weights spaced $T_{s}$ apart. The coefficients form the $(M+1) \times 1$ vector $\boldsymbol{w}(n)$, defined as $\boldsymbol{w}(n)=\left[w_{0}(n), w_{1}(n), \ldots, w_{M}(n)\right]^{\prime}$. The FSE output is sampled at the chip rate and despread with the locally generated PN sequence of the desired user to obtain the chip estimate. The parameter $j^{-n}$ in the despreader keeps the signal constellation of the desired user on the real axis, as the MSK constellation is known to jump between real and imaginary axes every $T_{c}$ seconds [20]. The despreader output is therefore expressed as

$$
\tilde{b}_{n}^{(0)}=a_{n}^{(0)} j^{-n}\left[\boldsymbol{w}^{H}(n) \boldsymbol{x}(n)\right] .
$$

The FSE tap weights are updated by an adaptive algorithm, where, depending on the receiver type (SL or WL), the error signal used to correct the tap weights differs. Next, the simple least-mean square (LMS) algorithm for the two receivers is discussed.

\section{B. Adaptive Algorithm}

The iterative equation of the LMS algorithm [28] can be expressed as

$$
\boldsymbol{w}(n+1)=\boldsymbol{w}(n)+\mu\left(j^{-n} a_{n}^{(0)}\right) \varepsilon^{*}(n) \boldsymbol{x}(n)
$$

where the step size $\mu$ is a small positive constant. The parameter $j^{-n} a_{n}^{(0)}$ rids the content of the FSE input from the PN sequence of the desired user at the time of updating, and adjusts the phase 
of the correction term in (29). For the implementation of the SL receiver, the error term $\varepsilon(n)$ takes the conventional form of

$$
\varepsilon_{\mathrm{SL}}(n)=b_{n}^{(0)}-\tilde{b}_{n}^{(0)}
$$

when the adaptive algorithm is under training and a replica of $b_{n}^{(0)}$ is available at the receiver side. When the SNR is high enough to make the outputs of the decision device reliable, the algorithm switches to the decision-directed (DD) mode and the error term in (30) becomes $\varepsilon_{\mathrm{SL}, \mathrm{DD}}(n)=\hat{b}_{n}^{(0)}-\tilde{b}_{n}^{(0)}$. For the implementation of the $\mathrm{WL}$ receiver, the error term is modified to

$$
\varepsilon_{\mathrm{WL}}(n)=b_{n}^{(0)}-\Re\left\{\tilde{b}_{n}^{(0)}\right\}
$$

in the training mode, and $\varepsilon_{\mathrm{WL}, \mathrm{DD}}(n)=\hat{b}_{n}^{(0)}-\Re\left\{\tilde{b}_{n}^{(0)}\right\}$ in the DD mode. The derivation of (31) can be found in [14]. It can also be intuitively argued that when the signal constellation lies on the real axis, the two filters in Fig. 1 become complex conjugates, i.e., $\psi_{n}(t)=\phi_{n}^{*}(t)$. Hence, the collective effect of the two filters can also be realized by only one filter with a real operator afterwards. Therefore, the output of the $\mathrm{WL}$ receiver must be taken after the real operator, as shown in Fig. 4.

Receiver complexity is determined by the number of FSE taps $M+1$, the adaptive algorithm, and the updating rate. Simulation results for several scenarios in the AWGN channel show that typically $M+1=10$ taps or less is sufficient for the FSE to deliver the maximum SNR. The choice of the adaptive algorithm depends on the application and affordable computational complexity. If, for instance, faster convergence rates are desirable, the recursive least-squares (RLS) algorithm can be employed. The updating rate is an adjustable design parameter. In Fig. 4, the FSE tap weights are updated every chip period $T_{c}$. To decrease the burden of computational complexity, the updating rate can be lowered to a bit period $T_{b}$, as in [19], at the expense of slower convergence.

It is emphasized that the proposed adaptive receiver is only one of several options to implement the WL receiver. Another implementation method is through actual frequency shifters, each followed by a filter, as illustrated in Fig. 3. However, good estimates of the parameters of the interfering users (such as chip delays $T_{k}$ and signal powers $P_{k}$ ) are required to construct the filters. The advantage of the proposed adaptive receiver is that it needs no a priori knowledge of such parameters, and can automatically estimate them. As another example, an alternative frequency-domain approach to the implementation is discussed in [29].

\section{NUMERICAL RESULTS}

Numerical results presented in this section achieve two goals. In the first set of results, the feasibility of the proposed adaptive receiver is demonstrated. It is shown that the maximum theoretical SNR can be achieved by the adaptive receiver. Next, the performance improvement achieved by the WL receiver over the SL receiver is quantified in various conditions. The results also confirm the previous discussions on performance analysis.

Fig. 5 presents a sample of training curves of the LMS algorithm for the SL and WL receivers in two cases where the interfering users are (a) symmetric, and (b) asymmetric. The modula-

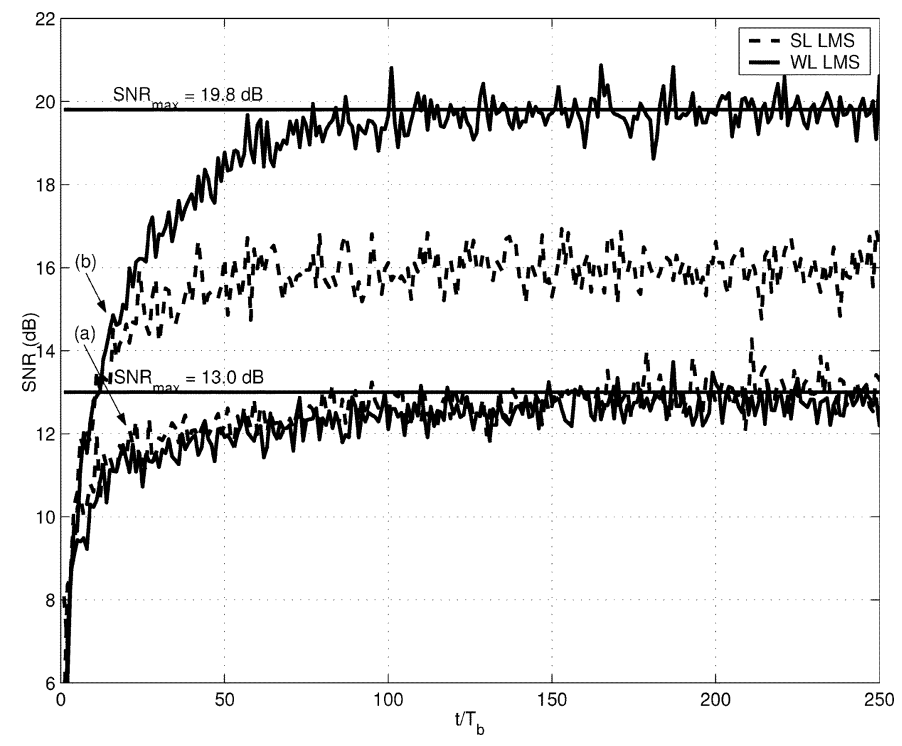

Fig. 5. Training curves for a system with three equally powered users. Solid and dashed curves represent WL-LMS and SL-LMS, respectively. Horizontal lines indicate the theoretical SNR $\max$. Chip delays of the interfering users are (a) $\boldsymbol{T}=\left[\begin{array}{ll}0.2 & 1.2\end{array}\right] T_{c}$ and (b) $\boldsymbol{T}=\left[\begin{array}{ll}0.6 & 0.8\end{array}\right] T_{c}$.

tion scheme is binary MSK, as described in Section V. It is noted that for MSK signals with half-cosine chip pulse, the period of CS is $T_{\mathrm{CS}}=2 T_{c}$. There are $K+1=3$ asynchronous equally powered users in the system with $E_{b} / \mathcal{N}_{0}=20 \mathrm{~dB}$. Other parameters are set as follows: $\mu=10^{-3}, N_{s}=2, N=16$, $M=15$, and $T_{D}=5 T_{c}$. Each curve is averaged over 100 Monte Carlo runs with the FSE tap weights initialized as $\boldsymbol{w}(0)=$ $\mathbf{0}_{(M+1) \times 1}$. In (a), the chip delays of the interfering users are $\boldsymbol{T}=\left[\begin{array}{ll}0.2 & 1.2\end{array}\right] T_{c}$, which according to (20) forms a symmetric set. It can be seen that both algorithms perform identically, and reach the $\mathrm{SNR}_{\max }=13.0 \mathrm{~dB}$. This is so, since MAI becomes proper and the WL receiver reduces to the SL receiver.In (b), the chip delays of the interfering users are $\boldsymbol{T}=\left[\begin{array}{ll}0.6 & 0.8\end{array}\right] T_{c}$, which makes MAI improper. Hence, the WL receiver significantly outperforms the SL receiver. The steady-state SNR of the WL algorithm is $19.8 \mathrm{~dB}$, whereas the SL algorithm stabilizes at $16.0 \mathrm{~dB}$.

Fig. 6 plots bit-error rate (BER) versus bit-energy-to-thermalnoise ratio $E_{b} / N_{0}$ under various network loads, with $K_{H}$ and $K_{L}$ representing the number of high- and low-powered users, respectively. The power of interferers in the $K_{H}$ set can be either 3 or $6 \mathrm{~dB}$ above the user of interest, whereas interferers in the $K_{L}$ set are of equal power with respect to the desired user. The power and delay profile of the active users in the network can be summarized in the $\boldsymbol{P}$ and $\boldsymbol{T}$ vectors, ${ }^{3}$ where $[\boldsymbol{P}]_{k, 1}=P_{k}$ and $[\boldsymbol{T}]_{k, 1}=T_{k}$. For cases (a) and (b), the first three and six elements of $\boldsymbol{P}$ and $\boldsymbol{T}$ are used, respectively. Also, $N=32$. Solid BER plots are simply obtained by invoking the standard Gaussian approximation (SGA) on the SNR. The points marked by " $\times$ " represent simulated BER when the receiver is implemented adaptively, as described in Fig. 5. It is observed that the adaptive implementation can achieve the predicted performance fairly accurately under various network loads. Moreover, com-

$$
\begin{aligned}
& \boldsymbol{P}=\left[\begin{array}{llllllllll}
2 & 4 & 1 & 1 & 2 & 2 & 1 & 1 & 2 & 2
\end{array}\right]^{\prime} P_{0} \\
& T=\left[\begin{array}{llllllllll}
0.6 & 0.8 & 0.5 & 0.2 & 0.7 & 0.1 & 0.3 & 0.4 & 0.1 & 0.25
\end{array}\right]^{\prime} T_{c}
\end{aligned}
$$




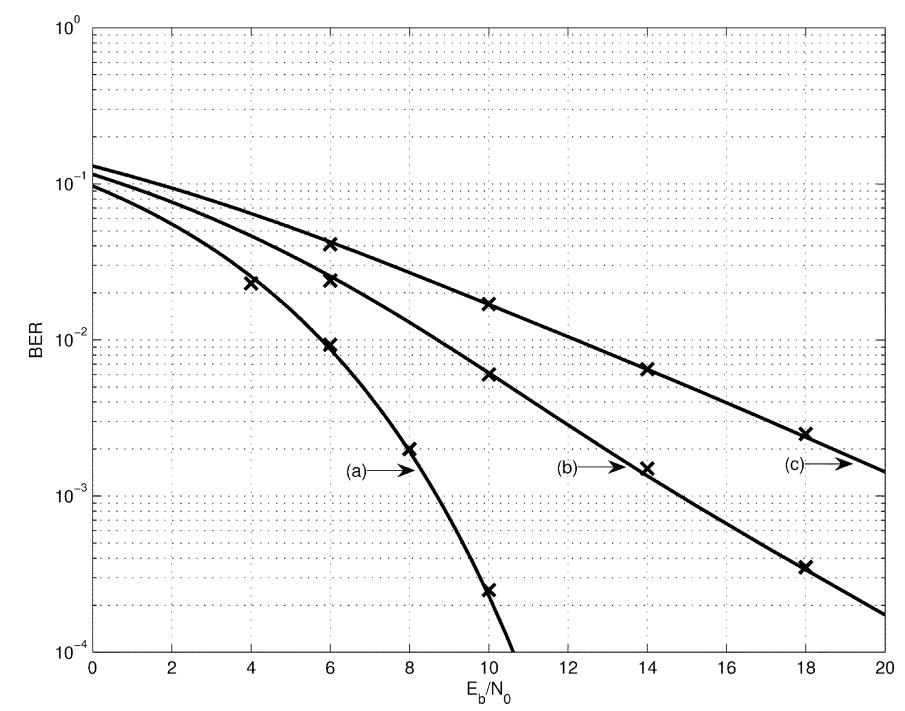

Fig. 6. BER versus $E_{b} / N_{0}$ for $N=32$ under diverse network loads. (a) $K_{H}=2$ and $K_{L}=1$. (b) $K_{H}=4$ and $K_{L}=2$. (c) $K_{H}=6$ and $K_{L}=4$. Solid lines represent the theoretical BER by invoking the SGA. Points marked by " $x$ " represent the simulation results when the receiver is implemented adaptively with $N_{s}=2$.

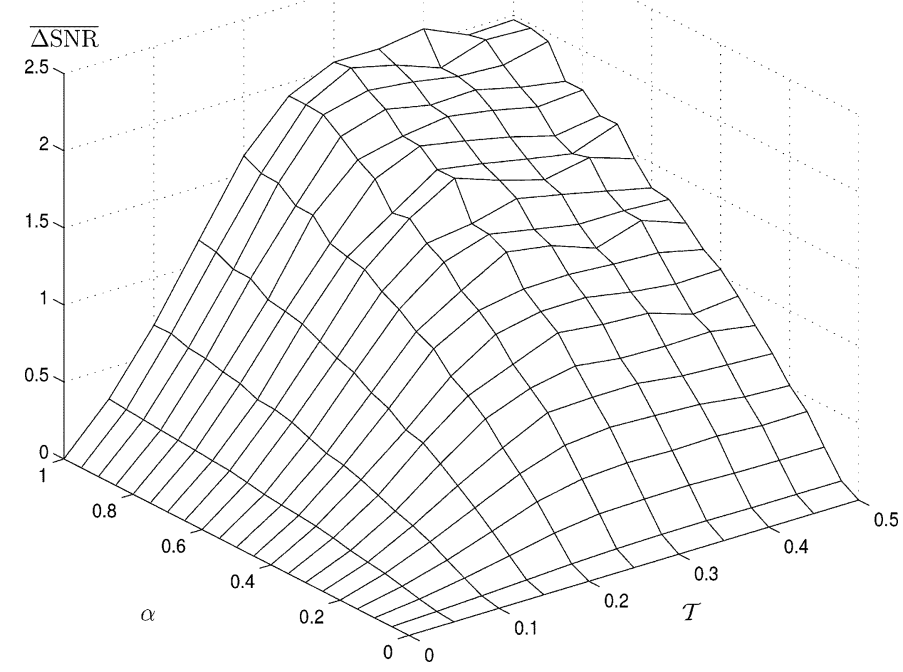

Fig. 7. $\overline{\Delta \mathrm{SNR}}$ versus $\alpha$ and $\mathcal{T}$ for a system with $K=3$ equally powered asynchronous users at $E_{b} / \mathcal{N}_{0}=20 \mathrm{~dB}$.

pared with plots (b) and (c), plot (a) shows a waterfall effect in $\mathrm{BER}$, confirming the near-far resistance of long-code MMSE receivers, where $K_{H}$ users can be effectively tuned out as long as $K_{H} \leq 1+\alpha$ [17], [30]. The remaining numerical results rely on evaluating (27) for performance comparison of the two receivers under the more spectrally efficient OQPSK modulation with Sqrt-RC pulse.

Fig. 7 plots $\overline{\Delta S N R}$, the average of $\Delta \mathrm{SNR}$, as a function of $\mathcal{T}$ and $\alpha$ when $0 \leq \mathcal{T} \leq 0.5 T_{c}$ and $0 \leq \alpha \leq 1$. There are $K+1=4$ asynchronous equally powered users in the system with $E_{b} / \mathcal{N}_{0}=20 \mathrm{~dB}$. The average is calculated over more than $10^{3}$ profiles of chip delays generated according to the uniform

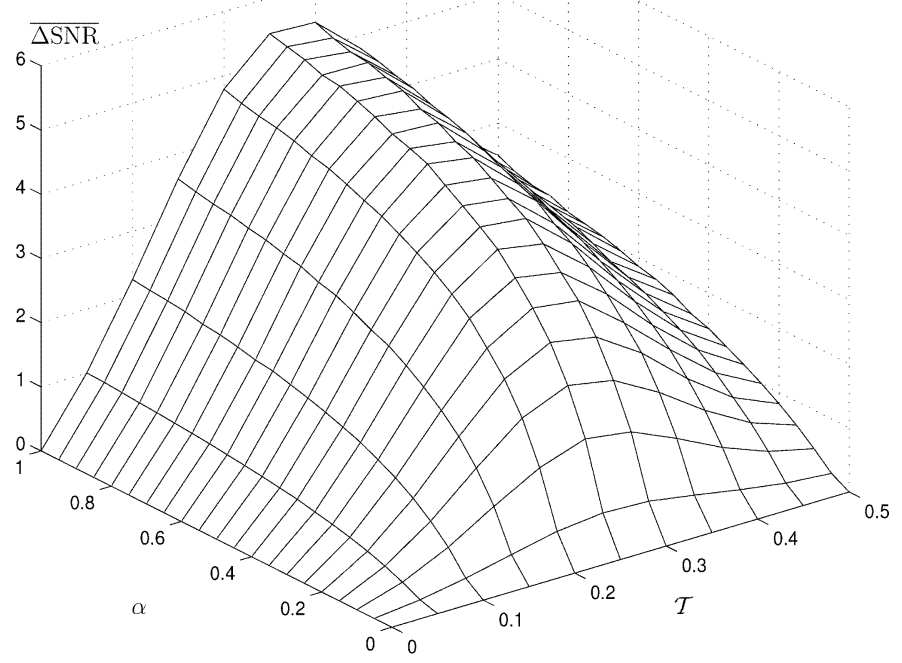

Fig. 8. $\overline{\Delta \mathrm{SNR}}$ versus $\alpha$ and $\mathcal{T}$ for a system with $K=30$ equally powered quasi-synchronous users, with $E_{b} / \mathcal{N}_{0}=20 \mathrm{~dB}$ and $T_{k} \in U\left[0,0.5 T_{c}\right)$.

distribution of $T_{k} \in U\left[0, T_{c}\right)$ to obtain tight confidence intervals. Upper and lower bounds of the $99 \%$ confidence interval differ by only $0.1 \mathrm{~dB}$. This is also true for the remaining figures of this section. The $\alpha$ axis can be interpreted as a measure of CS of the noise (MAI), whereas the $\mathcal{T}$ axis conceives the significance of improperness. Fig. 7 shows that as the noise approaches a WSS process $(\alpha \rightarrow 0)$ or becomes proper $(\mathcal{T} \rightarrow 0)$, the difference between the performance of the WL and SL receivers disappear. For $\mathcal{T} \neq 0$ and $\alpha \neq 0$, however, the WL receiver exploits the complementary autocorrelation function, which is overlooked in the SL receiver. As $\alpha \rightarrow 1$, the performance improvement grows. For instance, $\overline{\Delta \mathrm{SNR}}=0.6 \mathrm{~dB}$ for $(\alpha, \mathcal{T})=\left(0.22,0.5 T_{c}\right)$ and grows to slightly less than $2 \mathrm{~dB}$ for $(\alpha, \mathcal{T})=\left(1,0.5 T_{c}\right)$. Increasing the number of asynchronous equally powered users significantly decreases the performance improvement achieved by the WL receiver. It was observed that for $K=30, \overline{\Delta \mathrm{SNR}}$ is just $0.2 \mathrm{~dB}$ for $(\alpha, \mathcal{T})=\left(0.22,0.5 T_{c}\right)$, and approximately $0.5 \mathrm{~dB}$ for $(\alpha, \mathcal{T})=\left(1,0.5 T_{c}\right)$. Such decrease in $\overline{\triangle \mathrm{SNR}}$ is due to the fact that $\mathbb{S} \rightarrow 0$ with increasing $K$, as previously discussed.

Fig. 8 examines the effect of quasi-synchronous users on the performance improvement of the WL receiver. Here, the number of interfering users is $K=30$. However, users are quasi-synchronous, with chip delays chosen from the uniform distribution of $T_{k} \in U\left[0,0.5 T_{c}\right)$. Significant gains in the performance of the WL receiver can be observed even as $K=30$. The maximum value of $\overline{\Delta \text { SNR }}$ for each $\alpha$ appears around $\mathcal{T}=0.25 T_{c}$, with the peak of $\overline{\Delta \mathrm{SNR}}=5.5 \mathrm{~dB}$ in $(\alpha, \mathcal{T})=\left(1,0.25 T_{c}\right)$. Also for $(\alpha, \mathcal{T})=\left(0.22,0.5 T_{c}\right), \overline{\Delta \mathrm{SNR}}$ is slightly less than $1.0 \mathrm{~dB}$, compared with $0.2 \mathrm{~dB}$ in the asynchronous case.

The last set of numerical results investigates the presence of high-powered users in the system. In Fig. 9, the parameter setting is as that in Fig. 7, except that $P_{1}=2 P_{0}, P_{2}=4 P_{0}$, and $P_{3}=8 P_{0}$. Compared with Fig. 7 , the performance gain is now noticeably higher. For instance, at $(\alpha, \mathcal{T})=\left(0.22,0.5 T_{c}\right)$, $\overline{\Delta \mathrm{SNR}}$ grows to $1.7 \mathrm{~dB}$ from $0.6 \mathrm{~dB}$ in Fig. 7. The numerical results verify that (quasi-)synchronism and nonuniform power 


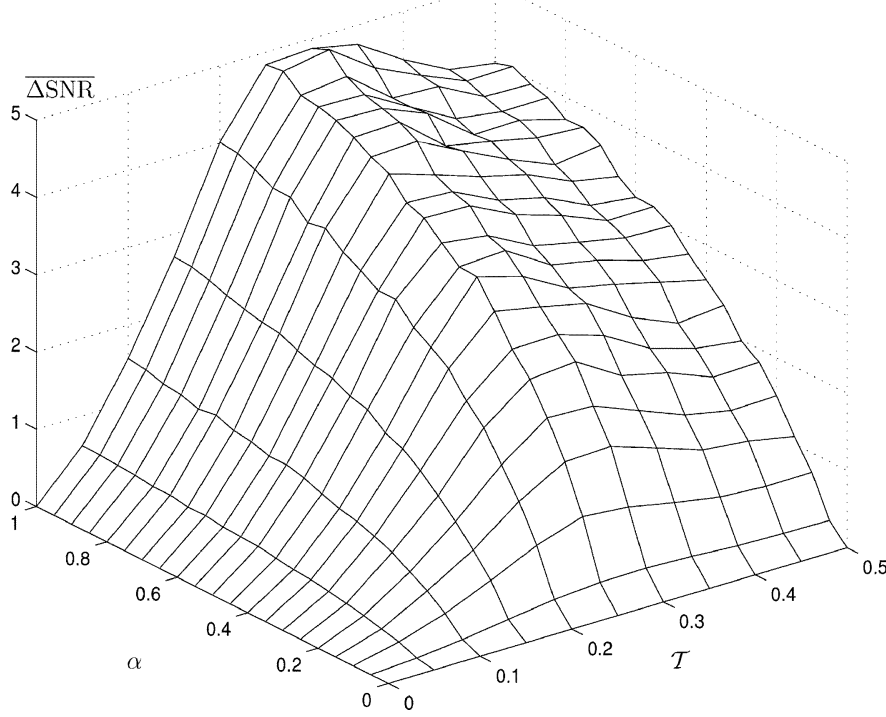

Fig. 9. $\overline{\triangle \mathrm{SNR}}$ versus $\alpha$ and $\mathcal{T}$ for a system with $K=3$ asynchronous users, with $P_{1}=2 P_{0}, P_{2}=4 P_{0}$, and $P_{3}=8 P_{0}$.

distribution are system features that drive the performance gain of the WL receiver over the SL receiver significantly higher.

\section{CONCLUSION}

This paper studied an enhanced WL receiver for CDMA systems when an OQPSK (or MSK) modulation scheme is employed. OQPSK modulation introduces improper complex noise, which necessitates the use of WL receivers instead of classical SL receivers. The design of WL receivers accounts for the generally nonzero complementary autocorrelation function that is overlooked in SL receivers. The distinguishing feature of this paper is its close look at the CS of bandlimited CDMA signals. The proposed receiver exploits the CS of the received signal through the excess bandwidth of the chip pulse by frequency-shifting its spectrum. The frequency response of the WL receiver was derived. Cases where the WL receiver reduces to the SL receiver were examined. An example of WL receiver implementation by adaptive algorithms was also proposed and verified to deliver the SNR performance predicted by theory. Moreover, scenarios where the WL receiver outperforms the SL receiver were identified. It was shown that (quasi-)synchronous systems with high-powered users, that can occur in future generation systems, yield remarkable gains in SNR performance. However, in asynchronous and quasi-synchronous transmission modes, performance gain of the WL receiver degrades unless the number of high-powered active users remains small. It has been shown in the literature that even the SL receiver has such a limitation. Further research is required to find how WL processing can be effectively applied to fully loaded systems.

\section{APPENDIX I}

PROOF OF $\alpha_{n}=\beta_{n}$

To prove the equality of the two scalars, it will be shown that $\alpha_{n}$ and $\beta_{n}$ are directly related to SNR in the I and Q components, respectively.
The desired signal in the I component is

$$
\begin{array}{r}
S_{n}^{\mathrm{WL}, \mathrm{I}}=\sqrt{\frac{P_{0}}{2}} b_{n}^{(\mathrm{I}, 0)} \int_{-\infty}^{\infty} a_{n}^{(\mathrm{I}, 0)}\left(u-n T_{b}\right) \phi_{n}\left(T_{b}-u\right) \\
+a_{n}^{(\mathrm{I}, 0)}\left(u-n T_{b}\right) \psi_{n}\left(T_{b}-u\right) d u .
\end{array}
$$

To obtain the variance of noise in (8), passband representation of signals and filters in terms of their baseband forms is used. For instance, the passband form of the noise signal can be expressed as $N(t)=(1 / 2)\left[n(t) e^{j \omega_{c} t}+n^{*}(t) e^{-j \omega_{c} t}\right]$, where $\omega_{c}$ is the carrier frequency [23]. Following the same steps as in [8] and after simplifications, the noise variance can be expressed as

$$
\begin{aligned}
\left(\sigma_{n}^{\mathrm{WL}}\right)^{2}=\int_{-\infty}^{\infty} a_{n}^{(\mathrm{I}, 0)}( & \left.u-n T_{b}\right) \phi_{n}\left(T_{b}-u\right) \\
& +a_{n}^{(\mathrm{I}, 0)}\left(u-n T_{b}\right) \psi_{n}\left(T_{b}-u\right) d u .
\end{aligned}
$$

The signal components in the I and Q phases are i.i.d., and hence, it can be shown that $S_{n}^{\mathrm{WL}, \mathrm{I}}=S_{n}^{\mathrm{WL}, \mathrm{Q}}=0.5 S_{n}^{\mathrm{WL}}$, where $S_{n}^{\mathrm{WL}}$ is the overall desired signal and $\left(\sigma_{n}^{\mathrm{WL}, \mathrm{I}}\right)^{2}=$ $\left(\sigma_{n}^{\mathrm{WL}, \mathrm{Q}}\right)^{2}=0.5\left(\sigma_{n}^{\mathrm{WL}}\right)^{2}$. Hence, it is straightforward to show that $\mathrm{SNR}_{n}^{\mathrm{WL}, \mathrm{I}}=\mathrm{SNR}_{n}^{\mathrm{WL}, \mathrm{Q}}=0.5 \mathrm{SNR}_{n}^{\mathrm{WL}}$. From (32) and (33), $\alpha_{n}$ can be expressed as $\alpha_{n}=\sqrt{P_{0} / 2}-\mathrm{SNR}_{n}^{\mathrm{WL}, \mathrm{I}}$. Similarly, $\beta_{n}$ can be written as $\beta_{n}=\sqrt{P_{0} / 2}-\mathrm{SNR}_{n}^{\mathrm{WL}, \mathrm{Q}}$, and consequently, $\alpha_{n}=\beta_{n}$.

\section{APPENDIX II \\ ELEMENTS OF THE MATRIX EQUATIONS}

Using the HSR technique, the frequency response of $g_{n}(t)$, $G_{n}(f)$, can be written as

$$
G_{n}(f)=\sum_{m=-M_{H}}^{M_{H}} G_{n, m}\left(f-\frac{m}{T_{c}}\right)
$$

where $M_{H}=\lceil\alpha / 2\rceil, G_{n, m}(f)=V(f) G_{n}\left(f+m / T_{c}\right)$, and with $V(f)=1$ if $|f| \leq 1 /\left(2 T_{c}\right)$ and $V(f)=0$, otherwise. The vector $\mathbb{G}_{n}$ is an $L_{H} \times 1$ column vector where $L_{H}=2 M_{H}+1$. Each of its arrays consists of a portion of $G_{n}(f)$ frequency response shifted to the $|f| \leq 1 /\left(2 T_{c}\right)$ band and filtered by an ideal low-pass filter. More specifically, $\left[\mathbb{G}_{n}\right]_{i, 1}=G_{n, i+1-L_{H}}(f)$. The $L_{H} \times 1$ column vector of $\mathbb{H}_{n}$ is constructed from $H_{n}(f)$, the frequency response of $h_{n}(t)$, in a similar fashion.

The vectors on the RHS of the matrix equations are also $L_{H} \times$ 1 vectors defined as

$$
\begin{aligned}
& \mathbb{Q}_{n}^{+}=\left[\mathcal{A}_{n}^{(\mathrm{I}, 0)}\left(e^{j 2 \pi f T_{c}}\right)+\mathcal{A}_{n}^{(\mathrm{Q}, 0)}\left(e^{j 2 \pi f T_{c}}\right)\right] e^{-j 2 \pi f T_{b}} \mathbb{Q} \mathbb{U} \\
& \mathbb{Q}_{n}^{-}=\left[\mathcal{A}_{n}^{(\mathrm{I}, 0)}\left(e^{j 2 \pi f T_{c}}\right)-\mathcal{A}_{n}^{(\mathrm{Q}, 0)}\left(e^{j 2 \pi f T_{c}}\right)\right] e^{-j 2 \pi f T_{b}} \mathbb{Q} \mathbb{U}
\end{aligned}
$$

where

$$
\begin{aligned}
\mathcal{A}_{n}^{(\mathrm{I}, 0)}(z) & =\sum_{i=n N}^{(n+1) N-1} a_{i+n N}^{(\mathrm{I}, 0)} z^{-i} \\
\mathcal{A}_{n}^{(\mathrm{Q}, 0)}(z) & =\sum_{i=n N}^{(n+1) N-1} a_{i+n N}^{(\mathrm{I}, 0)} z^{-\left(i+\mathcal{T} / T_{c}\right)}
\end{aligned}
$$

are the $Z$-transforms of the desired user's spreading sequences $\boldsymbol{a}_{n}^{(\mathrm{I}, k)}$ and $\boldsymbol{a}_{n}^{(\mathrm{Q}, k)}$, with the effect of time offset in the Q phase properly introduced in (36b). The matrix $\mathbb{Q}$ is an $L_{H} \times L_{H}$ 
diagonal matrix containing partial bands of $Q(f)$, the frequency response of $q(t)$, where the nonzero arrays are defined as $[\mathbb{Q}]_{i, i}=Q_{i+1-L_{H}}(f)$. The $L_{H} \times 1$ column vector $\mathbb{U}$ is defined as $[U]_{i, 1}=1$.

The matrix $\mathbb{R}$ representing the cross spectral density (CSD) of the noise is

$$
\mathbb{R}=\mathcal{N}_{0} \mathbb{q}+\frac{1}{T_{c}} \mathbb{Q} \mathbb{P}^{\mathbb{R}} \mathbb{Q}
$$

where $\square$ is the $L_{H} \times L_{H}$ identity matrix. All the information concerning the chip delays and signal powers of interfering users is contained in the power matrix

$$
\mathbb{P}^{\mathbb{R}}=\sum_{k=1}^{K} \boldsymbol{P}_{k, T_{k}} \boldsymbol{P}_{k, T_{k}}^{H}+\boldsymbol{P}_{k, T_{k}+\mathcal{T}} \boldsymbol{P}_{k, T_{k}+\mathcal{T}}^{H}
$$

where $H$ is the Hermitian transpose, and the $L_{H} \times 1$ column vector $\boldsymbol{P}_{k, t_{0}}$ is

$$
\left[\boldsymbol{P}_{k, t_{0}}\right]_{i, 1}=\sqrt{\frac{P_{k}}{2}} \exp \left(\frac{-j 2 \pi\left(i+1-L_{H}\right) t_{0}}{T_{c}}\right) .
$$

The matrix $\mathbb{S}$ representing the HSR of the complementary autocorrelation function is formulated as

$$
\mathbb{S}=\frac{1}{T_{c}} \mathbb{Q} \mathbb{P}^{\mathbb{S}} \mathbb{Q}
$$

where

$$
\mathbb{P}^{\mathbb{S}}=\sum_{k=1}^{K} \boldsymbol{P}_{k, T_{k}} \boldsymbol{P}_{k, T_{k}}^{\prime}-\boldsymbol{P}_{k, T_{k}+\mathcal{T}} \boldsymbol{P}_{k, T_{k}+\mathcal{T}}^{\prime}
$$

with ' denoting the transpose operator.

\section{ACKNOWLEDGMENT}

The authors would like to thank the anonymous reviewers for their contructive comments and suggestions which led to a significant improvement of the manuscript.

\section{REFERENCES}

[1] W. M. Brown and R. B. Crane, "Conjugate linear filtering," IEEE Trans. Inf. Theory, vol. IT-15, no. 7, pp. 462-465, Jul. 1969.

[2] B. Picinbono and P. Chevalier, "Widely linear estimation with complex data," IEEE Trans. Signal Process., vol. 43, no. 8, pp. 2030-2033, Aug. 1995.

[3] F. D. Neeser and J. L. Massey, "Proper complex random processes with applications to information theory," IEEE Trans. Inf. Theory, vol. 39, no. 7, pp. 1293-1302, Jul. 1993.

[4] S. M. Alamouti, "A simple transmit diversity technique for wireless communications," IEEE Trans. Commun., vol. 16, no. 10, pp. 1451-1458, Oct. 1998.

[5] E. A. Lee and D. G. Messerschmitt, Digital Communication, 2nd ed. Norwell, MA: Kluwer, 1994.

[6] Mobile Station-Base Station Compatibility Standard for Dual-Mode Wideband Spread Spectrum Cellular Systems, TIA/EIA/IS-95, 1993.

[7] Physical Layer Standard for cdma2000 Spread Spectrum Systems (2002, May). [Online]. Available: http://www.3gpp2.org. Tech. Steering Group $\mathrm{C}$ (TSG-C)

[8] Y. C. Yoon and H. Leib, "Maximizing SNR in improper complex noise and applications to CDMA," IEEE Commun. Lett., vol. 1, no. 1, pp. 5-8, Jan. 1997.

[9] S. Buzzi, M. Lops, and A. M. Tulino, "A new family of MMSE multiuser receivers for interference suppression in DS/CDMA systems employing BPSK modulation," IEEE Trans. Commun., vol. 49, no. 1, pp. 154-167, Jan. 2001.
[10] S. Buzzi and M. Lops, "Performance analysis for the improved linear multiuser detectors in BPSK-modulated DS-CDMA systems," IEEE Trans. Commun., vol. 51, no. 1, pp. 37-42, Jan. 2003.

[11] G. Gelli, L. Paura, and R. P. Ragozini, "Blind widely linear multiuser detection," IEEE Commun. Lett., vol. 4, no. 6, pp. 187-189, Jun. 2000.

[12] S. Buzzi, M. Lops, and A. M. Tulino, "A generalized minimum-meanoutput-energy strategy for CDMA systems with improper MAI," IEEE Trans. Inf. Theory, vol. 48, no. 3, pp. 761-767, Mar. 2002.

[13] R. Schober, W. H. Gerstacker, and L. Lampe, "A blind widely linear minimum-output-energy algorithm," in Proc. IEEE Wireless Commun. Netw. Conf., vol. 1, 2003, pp. 612-617.

[14] _ "A widely linear LMS algorithm for MAI suppression in DS-CDMA," in Proc. IEEE Int. Conf. Commun., vol. 4, 2003, pp. $2520-2525$.

[15] W. H. Gerstacker, F. Obernosterer, R. Schober, A. Lehmann, A. Lampe, and P. Gunreben, "Widely linear equalization for space-time block-coded transmission over fading ISI channels," in Proc. IEEE Veh Technol. Conf., vol. 1, Sep. 2002, pp. 238-242.

[16] W. H. Gerstacker, R. Schober, and A. Lampe, "Receivers with widely linear processing for frequency-selective channels," IEEE Trans. Commun., vol. 51, no. 9, pp. 1512-1523, Sep. 2003.

[17] T. F. Wong, T. M. Lok, and J. S. Lehnert, "Asynchronous multiple-access interference suppression and chip waveform selection with aperiodic random sequences," IEEE Trans. Commun., vol. 47, no. 1, pp. 103-114, Jan. 1999

[18] W. A. Gardner, "Cyclic Wiener filtering: Theory and method," IEEE Trans. Commun., vol. 41, no. 1, pp. 151-163, Jan. 1993.

[19] A. Mirbagheri and Y. C. Yoon, "A linear MMSE receiver for multipath asynchronous random-CDMA with chip pulse shaping," IEEE Trans. Veh. Technol., vol. 51, no. 5, pp. 1072-1086, Sep. 2002.

[20] S. Pasupathy, "Minimum shift keying: A spectrally efficient modulation," IEEE Commun. Mag., vol. 17, no. 4, pp. 14-22, Jul. 1979.

[21] Y. C. Yoon and H. Leib, "Chip-delay locked matched filter for DS-CDMA systems using long sequence spreading," IEEE Trans. Commun., vol. 49, no. 8, pp. 1468-1478, Aug. 2001.

[22] L. E. Franks, "Polyperiodic linear filtering," in Cyclostationarity in Communications and Signal Processing, W. A. Gardner, Ed. New York: IEEE Press, 1994, pp. 240-266.

[23] J. G. Proakis, Digital Communications, 3rd ed. New York: McGrawHill, 1995

[24] P. J. Schreier and L. L. Scharf, "Second-order analysis of improper complex random vectors and processes," IEEE Trans. Signal Process., vol. 51, no. 3, pp. 714-725, Mar. 2003.

[25] V. M. DaSilva and E. S. Sousa, "Multicarrier orthogonal CDMA signals for quasi-synchronous communication systems," IEEE J. Sel. Areas Commun., vol. 12, no. 5, pp. 842-852, Jun. 1994.

[26] T. K. Woo, "Orthogonal code design for quasisynchronous CDMA," IEE Electron. Lett., vol. 36, no. 19, pp. 1632-1633, Sep. 2000.

[27] W. A. Gardner and W. A. Brown, "Frequency-shift filtering theory for adaptive co-channel interference removal," in Proc. Asilomar Conf. Signals, Syst., Comput., Pacific Grove, CA, Oct. 1989, pp. 562-567.

[28] S. Haykin, Adaptive Filter Theory, 3rd ed. Englewood Cliffs, NJ: Prentice-Hall, 1996.

[29] E. R. Ferrara, "Frequency-domain implementations of periodically time-varying filters," IEEE Trans. Acoust., Speech, Signal Process., vol. ASSP-33, no. 4, pp. 883-892, Aug. 1985.

[30] Y. C. Yoon, "SNR maximizing linear filters with interference suppression capabilities for DS-CDMA," Ph.D. dissertation, McGill Univ., Montreal, QC, Canada, 1998.

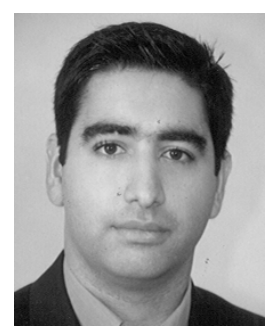

Arash Mirbagheri (S'96-M'04) was born in Tehran, Iran. He received the B.Sc. degree in electrical engineering from Sharif University of Technology, Tehran, Iran, in 1996, and the Ph.D. degree in electrical and computer engineering from the University of Waterloo, Waterloo, ON, Canada in 2003

From September 2003 to August 2004, he was a Postdoctoral Fellow with the Department of Electrical and Computer Engineering, University of Toronto, Toronto, ON, Canada. He is presently with Qualcomm CDMA Technologies, Qualcomm Inc., San Diego, CA. His current research activities are in the areas of code-division multiple access (CDMA), orthogonal frequency-division multiplexing (OFDM), and the applications of adaptive filtering theory in digital and wireless communication systems.

Dr. Mirbagheri was the recipient of the Natural Sciences and Engineering Research Council (NSERC) of Canada postgraduate scholarship, the Canadian Wireless Telecommunications Association (CWTA) graduate scholarship, and the Ontario Graduate Scholarship for Science and Technology. 


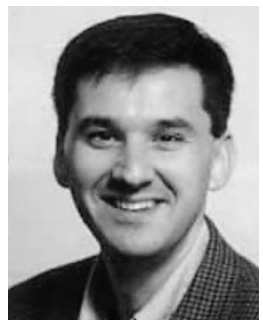

Konstantinos N. Plataniotis (S'90-M'92-SM'03) received the $\mathrm{B}$. Eng. degree in computer engineering from the University of Patras, Patras, Greece in 1988, and the M.S. and Ph.D. degrees in electrical engineering from the Florida Institute of Technology (Florida Tech), Melbourne, FL, in 1992 and 1994, respectively.

He is an Associate Professor with The Edward S. Rogers Sr. Department of Electrical and Computer Engineering, University of Toronto, Toronto, ON, Canada. His research interests include signal and image processing, communications systems, biometrics, stochastic estimation, and pattern recognition.

Dr. Plataniotis is a registered Professional Engineer in the Province of Ontario, and a member of the Technical Chamber of Greece. He is an Associate Editor for the IEEE TRANSACTIONS ON NEURAL NETwORKS, the Technical Program Co-Chair for the IEEE International Conference on Multimedia and Expo (ICME) 2006, and the Vice-Chair of the IEEE Intelligent Transportation Systems Conference (ITSC) 2006. He is the 2005 recipient of IEEE Canada's Outstanding Engineering Educator Award "for contributions to engineering education and inspirational guidance of graduate students."

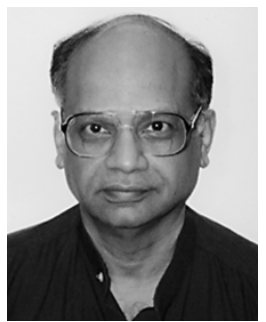

Subbarayan Pasupathy (M'73-SM'81-LF'91) was born in Chennai (Madras), India, on September 21, 1940. He received the B.E. degree in telecommunications from the University of Madras, Madras, India, in 1963, the M.Tech. degree in electrical engineering from the Indian Institute of Technology, Madras, India, in 1966, and the M.Phil. and Ph.D. degrees in engineering and applied science from Yale University, New Haven, CT, in 1970 and 1972, respectively.

He joined the faculty of the University of Toronto, Toronto, ON, Canada, in 1973 and became a Professor of Electrical Engineering in 1983. He has served as Chairman of the Communications Group and as the Associate Chairman of the Department of Electrical Engineering at the University of Toronto. His research interests are in the areas of communications theory, digital communications, and statistical signal processing.

Dr. Pasupathy is a Registered Professional Engineer in the Province of Ontario. He was awarded the Canadian Award in Telecommunications in 2003 by the Canadian Society of Information Theory, and was elected as a Fellow of the Engineering Society of Canada in 2004. He has served as a Technical Associate Editor for the IEEE Communications Magazine (1979-1982) and as an Associate Editor for the Canadian Electrical Engineering Journal (1980-1983). During 1982-1989, he was an Area Editor for Data Communications and Modulation for the IEEE TRANSACTIONS ON COMMUNICATIONS. From 1984 to 1998, he wrote a regular column entitled "Light Traffic" for the IEEE Communications Magazine. 